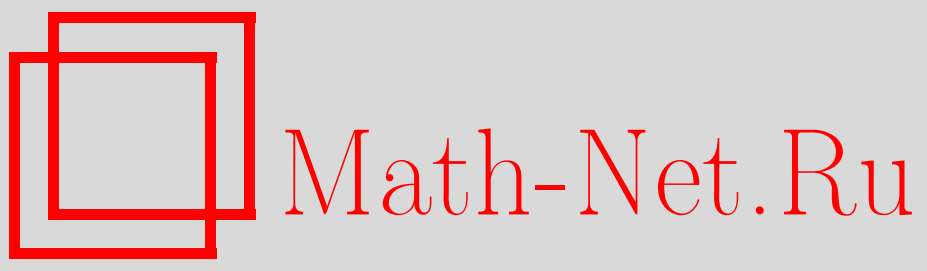

А. М. Бородин, Характеры симметрических групп и корреляционные функции точечных процессов, Функи. анализ и его прил., 2000, том 34, выпуск $1,12-28$

DOI: https://doi.org/10.4213/faa279

Использование Общероссийского математического портала MathNet.Ru подразумевает, что вы прочитали и согласны с пользовательским соглашением

http://www . mathnet.ru/rus/agreement

Параметры загрузки:

IP : 52.205 .19 .152

26 апреля 2023 г., 11:29:00

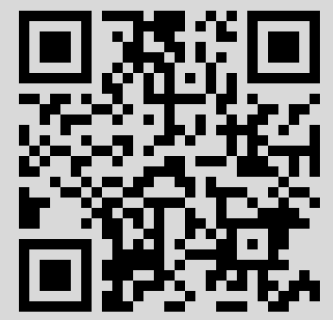




\title{
Характеры симметрических групп и корреляционные функции точечных процессов*
}

\author{
(c) 2000. А. М. Бородин
}

\section{$\S 0$. Введение}

Пусть $S(\infty)$ - группа финитных перестановок натурального ряда и $\chi-$ некоторая положительно определенная центральная функция на ней, нормированная так, что $\chi(e)=1$. Сужение $\chi_{n}$ функции $\chi$ на конечную симметрическую группу $S_{n} \subset S(\infty)$ является положительно определенной центральной функцией на $S_{n}$. Задача, которую мы решаем в этой работе, может быть сформулирована следующим образом: как описать функцию $\chi$, если известны преобразования Фурье всех функций $\chi_{n}$ ?

Этот вопрос мотивирован теорией представлений. Центральные положительно определенные нормированные функции на $S(\infty)$ находятся во взаимно однозначном соответствии со сферическими представлениями пары Гельфанда $(G, K)$, где $G=S(\infty) \times S(\infty)$, а $K$ - диагональная подгруппа группы $G$ (см. [8]). Ясно, что такие функции образуют выпуклое множество, причем его крайние точки соответствуют неприводимым представлениям. Знание преобразований Фурье функций $\chi_{n}$ - это в точности знание того, как соответствующее представление после сужения на $\left(S_{n} \times S_{n}, \operatorname{diag} S_{n}\right)$ разлагается на неприводимые. Нас же интересует, как разлагается на неприводимые исходное представление.

Впервые этот вопрос возник в работе [6], где рассматривались так называемые обобщенные регулярные представления пары $(G, K)$. Подход, излагаемый в настоящей работе, позволил полностью решить задачу о разложении обобщенных регулярных представлений на неприводимые и показать ее тесную связь с теорией случайных матриц $[9-13,1]$.

Неприводимые сферические представления пары $(G, K)$ (или крайние точки множества центральных положительно определенных нормированных функций на $S(\infty))$ параметризуются точками симплекса Тома́ (см. [14])

$$
\Omega=\left\{\alpha_{1} \geqslant \alpha_{2} \geqslant \ldots \geqslant 0, \beta_{1} \geqslant \beta_{2} \geqslant \ldots \geqslant 0, \sum_{i=1}^{\infty}\left(\alpha_{i}+\beta_{i}\right) \leqslant 1\right\} .
$$

Всякая центральная положительно определенная нормированная функция $\chi$ на $S(\infty)$ представляется единственным образом как выпуклая комбинация крайних функций:

$$
\chi=\int_{\Omega} \chi_{\alpha, \beta} P_{\chi}(d(\alpha, \beta)),
$$

где $P_{\chi}$ - некоторая вероятностная мера на $\Omega$.

* Исследования поддержаны Российской программой поддержки научных школ (грант 96-15-96060). 
Коэффициенты Фурье функций $\chi_{n}$ являются (см. [3]) интегралами по $\Omega$ так называемых расширенных функиий Шура относительно меры $P_{\chi}$. Нашей целью будет описание меры $P_{\chi}$ через эти интегралы.

Г. И. Ольшанский предложил связать с мерой $P_{\chi}$ случайный точечный процесс на $\mathbb{R}^{*}=\mathbb{R} \backslash\{0\}$ следующим образом (см. [9]). Поставим в соответствие каждой точке $(\alpha, \beta) \in \Omega$ точечную конфигурацию в $\mathbb{R}^{*}$ :

$$
(\alpha, \beta) \mapsto\left(\alpha_{1}, \alpha_{2}, \ldots,-\beta_{1},-\beta_{2}, \ldots\right) .
$$

Тогда $P_{\chi}$ становится мерой на пространстве точечных конфигураций или, согласно традиционной терминологии, случайным точечным процессом. В дальнейшем мы будем следовать этому подходу.

Основным результатом настоящей работы является метод вычисления корреляционных функций случайного процесса, ассоциированного с $\chi$, через коэффициенты Фурье функций $\chi_{n}$. Метод включает решение некоторых проблем моментов на $\mathbb{R}^{d}, d=1,2, \ldots$.

С алгебраической точки зрения переход от интегралов расширенных функций Шура к некоторым аналогам моментов корреляционных функций тесно связан с переходом от базиса функций Шура к базису сумм Ньютона в алгебре симметрических функций. Соответствующая матрица перехода состоит из значений характеров конечных симметрических групп. Основная трудность состоит в том, что для этих значений нет явных формул. Все, чем мы располагаем, - это некая рекуррентная вычислительная процедура (правило МурнаганаНакаямы), которую мы и будем использовать.

Оказывается, что наш подход работает не только для мер на $\Omega$, но и для мер на несколько большем пространстве $\widetilde{\Omega}$ (определение см. ниже). Поскольку это обобщение имеет важные приложения (см. [2]), мы будем работать на $\widetilde{\Omega}$.

Автор искренне благодарит Г. И. Ольшанского, без постоянного внимания и поддержки которого эта работа вряд ли была бы написана.

\section{§1. Предварительные сведения}

Обозначим через $\widetilde{\Omega}$ множество троек $\omega=(\alpha, \beta ; \tau)$, где $\alpha=\left(\alpha_{1} \geqslant \alpha_{2} \geqslant \cdots\right.$ $\geqslant 0), \beta=\left(\beta_{1} \geqslant \beta_{2} \geqslant \ldots \geqslant 0\right), \tau \in \mathbb{R}_{+}$и $\sum_{i=1}^{\infty}\left(\alpha_{i}+\beta_{i}\right) \leqslant \tau$. Мы снабжаем $\widetilde{\Omega}$ слабейшей топологией, в которой $\alpha_{i}, \beta_{i}$ и $\tau$ являются непрерывными функциями. В этой топологии $\widetilde{\Omega}$ локально компактно. Заметим, что $\widetilde{\Omega}$ гомеоморфно $\Omega \times \mathbb{R}_{+}$ по модулю отождествления множества $\Omega \times\{0\}$ с одной точкой $(0,0,0) \in \widetilde{\Omega}$; соответствующее отображение имеет вид

$$
((\alpha, \beta), \tau) \in \Omega \times \mathbb{R}_{+} \mapsto(\tau \alpha, \tau \beta, \tau) \in \widetilde{\Omega} .
$$

Определим вложение алгебры $\Lambda$ симметрических функций (ее определение можно найти в [7]) в алгебру $C(\widetilde{\Omega})$ непрерывных функций на $\widetilde{\Omega}$. Поскольку $\Lambda=\mathbb{C}\left[p_{1}, p_{2}, \ldots\right]$, где $p_{k}=\sum_{i} x_{i}^{k}$ — это суммы Ньютона, достаточно указать образы всех $p_{k}$. Положим их равными

$$
\tilde{p}_{k}(\omega)= \begin{cases}\tau, & k=1, \\ \sum_{i=1}^{\infty} \alpha_{i}^{k}-\sum_{i=1}^{\infty}\left(-\beta_{i}\right)^{k}, & k \geqslant 2 .\end{cases}
$$

Можно показать, что $\tilde{p}_{k}$ действительно лежат в $C(\widetilde{\Omega})$ (cм. [5, Lemma 5.2]). 
Образы функций Шура $\left\{s_{\lambda}\right\}$ (см. [7]) при этом вложении называются расширенныли функциями Шура и обозначаются $\left\{\tilde{s}_{\lambda}\right\}$ (ср. [3]). Эти функции неотрицательны (см. [3, 5]).

Из хорошо известного выражения функций Шура через суммы Ньютона $[7, \S$ I.7] следует, что

$$
\tilde{s}_{\lambda}=\sum_{\rho} \chi_{\rho}^{\lambda} z_{\rho}^{-1} \tilde{p}_{\rho},
$$

где $\lambda=\left(\lambda_{1} \geqslant \lambda_{2} \geqslant \ldots\right)$ - произвольное разбиение, сумма берется по всем разбиениям $\rho=\left(\rho_{1} \geqslant \rho_{2} \geqslant \ldots\right)$ числа $|\lambda|=\lambda_{1}+\lambda_{2}+\ldots, \chi^{\lambda}$ - неприводимый характер симметрической группы порядка $|\lambda|$, соответствующий разбиению $\lambda$, $\chi_{\rho}^{\lambda}$ - его значение на классе сопряженности $\rho$, причем $z_{\rho}^{-1}|\lambda|$ ! - мощность этого класса сопряженности, и $\tilde{p}_{\rho}=\tilde{p}_{\rho_{1}} \tilde{p}_{\rho_{2}} \cdots$.

Пусть $P$ - некоторая борелевская вероятностная мера на $\widetilde{\Omega}$, удовлетворяющая условию

$$
m_{k}=\int_{\widetilde{\Omega}} \tau^{k}(\omega) P(d \omega)<\infty, \quad k=0,1,2, \ldots
$$

Поскольку $\left|\tilde{p}_{k}(\omega)\right| \leqslant \tau^{k}(\omega)$, из $(1.1)$ следует, что $\tilde{s}_{\lambda}(\omega) \leqslant$ const $\cdot \tau^{|\lambda|}(\omega)$ и константа не зависит от $\omega$. Тогда условие (1.2) гарантирует конечность интегралов

$$
\phi(\lambda)=\int_{\widetilde{\Omega}} \tilde{s}_{\lambda}(\omega) P(d \omega)
$$

для всех разбиений $\lambda$.

Определим теперь точечный случайный процесс на проколотой действительной оси $\mathbb{R}^{*}=\mathbb{R} \backslash\{0\}$, ассоциированный с мерой $P$. Общая информация о точечных процессах может быть найдена в книге [4].

Всякой тройке $(\alpha, \beta, \tau) \in \widetilde{\Omega}$ мы ставим в соответствие точечную конфигурацию в $\mathbb{R}^{*}$ следующим образом:

$$
(\alpha, \beta, \tau) \mapsto\left(\alpha_{1}, \alpha_{2}, \ldots,-\beta_{1},-\beta_{2}, \ldots\right),
$$

где нули в $\alpha$ и $\beta$ мы опускаем. Единственная возможная предельная точка такой конфигурации - это нуль, так как $\lim _{k \rightarrow \infty} \alpha_{k}=\lim _{k \rightarrow \infty} \beta_{k}=0$.

Таким образом, наша мера $P$ становится вероятностной мерой на множестве таких точечных конфигураций, т. е. точечным процессом; мы будем обозначать этот процесс через $\mathscr{P}$.

Одним из способов описания точечных процессов является вычисление их коррелячионных мер ${ }^{1}$. В нашем случае $n$-я корреляционная мера живет на $\left(\mathbb{R}^{*}\right)^{n}$, и мы будем обозначать ее через $\rho_{n}(d x)$. Грубо говоря, $\rho_{n}\left(d x_{1} \cdots d x_{n}\right)$ - это вероятность найти точку случайной конфигурации в каждом из отрезков $\left[x_{i}, x_{i}+\right.$ $\left.d x_{i}\right], i=1, \ldots, n$.

ПРЕДЛОЖЕНИЕ 1.1 (Ольшанский [2]). Если мера $P$ удовлетворяет условию (1.2), то $n$-я корреляционная мера принимает конечные значения на всех множествах вида $(\mathbb{R} \backslash[-\varepsilon, \varepsilon])^{n}$ для любих $\varepsilon>0, n=1,2, \ldots$ Если, кроме того, для всех $n=1,2, \ldots$

\footnotetext{
${ }^{1} \mathrm{~B}$ книге [4] эти меры называются factorial moment measures.
} 


$$
\sum_{k=0}^{\infty}\left(m_{n+k}\right)^{-1 / k}=+\infty
$$

то коррелячионнье мерь однозначно определяют меру ЯР.

Мы будем всегда предполагать выполнение условия (1.4). В дальнейшем нам понадобится еще одно техническое условие для единственности решений некоторых проблем моментов. Мы будем предполагать, что существует такое $a>0$, что

$$
\int_{\widetilde{\Omega}} e^{a \tau(\omega)} P(d \omega)<\infty .
$$

Нам будет удобнее работать с плотностями корреляционных мер относительно меры Лебега; плотность $n$-й корреляционной меры называется $n$-й корреляиионной функиией и обозначается $\rho_{n}\left(x_{1}, \ldots, x_{n}\right)$. Таким образом, $\rho_{n}(x) d x=$ $\rho_{n}(d x)$. Как обобщенные функции $\rho_{n}\left(x_{1}, \ldots, x_{n}\right)$ всегда определены.

Зафиксируем класс обобщенных функций, с которым мы будем работать в дальнейшем.

Обозначим через $E_{n}(x)$ гладкую положительную функцию на $\mathbb{R}^{n}$, совпадающую с $e^{\sqrt{x_{1}^{2}+\cdots+x_{n}^{2}}}$ вне некоторого компактного множества. Обозначим через $\mathscr{V}_{k}^{a}, a>0$, векторное пространство линейных функционалов $F$ на пространстве финитных пробных функций от $k$ переменных, таких, что функционал $E_{k}(a x) \cdot F$ может быть продолжен до непрерывного функционала на пространстве Шварца $\mathscr{S}\left(\mathbb{R}^{k}\right)$. Мы будем рассматривать элементы из $\mathscr{V}_{k}^{a}$ как обобщенные функции вида $\frac{1}{E_{k}(a x)} \cdot G$, где $G \in \mathscr{S}^{\prime}\left(\mathbb{R}^{k}\right)$.

Заметим, что для всякого функционала $F \in \mathscr{V}_{k}^{a}$ корректно определены моменты $\left\langle F, x_{1}^{l_{1}} \cdots x_{k}^{l_{k}}\right\rangle, l_{i}=0,1, \ldots$, поскольку

$$
\frac{x_{1}^{l_{1}} \cdots x_{k}^{l_{k}}}{E_{k}(a x)} \in \mathscr{S}\left(\mathbb{R}^{k}\right) .
$$

Более того, элементы из $\mathscr{V}_{k}^{a}$ определяются своими моментами однозначно, так как линейные комбинации функций вида (1.6) образуют плотное подпространство в $\mathscr{S}\left(\mathbb{R}^{k}\right)$.

Ясно, что $\mathscr{V}_{k}^{a_{1}} \subset \mathscr{V}_{k}^{a_{2}}$, если $a_{1} \geqslant a_{2}$. Положим $\mathscr{V}_{k}=\bigcup_{a>0} \mathscr{V}_{k}^{a}$.

\section{$\S 2$. Основной результат}

Основной результат этой работы объясняет, как вычислить корреляционные функции точечного процесса $\mathscr{P}$, построенного выше, исходя из интегралов (1.3).

Для точной формулировки результата нам понадобятся некоторые обозначения.

Обозначим через $\Phi_{n, d}(n, d \in \mathbb{N})$ множество отображений

$$
\varphi:\{1, \ldots, n\} \rightarrow\left\{1,1^{\prime} ; \ldots ; d, d^{\prime}\right\}
$$

удовлетворяющих следующим условиям

1) $\varphi$ инъективно;

2) $\operatorname{Im} \varphi \cap\left\{m, m^{\prime}\right\} \neq \varnothing$ для всех $m=1, \ldots, d$. 
Ясно, что $\Phi_{n, d}$ непусто, если и только если $n / 2 \leqslant d \leqslant n$.

Для всякой (обобщенной) функции $F\left(r_{1}, s_{1} ; \ldots ; r_{d}, s_{d}\right)$ от $2 d$ переменных и $\varphi \in \Phi_{n, d}$ определим (обобщенную) функцию $(\varphi F)\left(x_{1}, \ldots, x_{n}\right)$ от $n$ переменных следующим образом. Произведем следующее переименование переменных: обозначим $r_{i}$ через $x_{k}$, если $\varphi(k)=i$, и обозначим $s_{j}$ через $x_{k}$, если $\varphi(k)=j^{\prime}$, причем проделаем это для всех $r_{i}, s_{j}$, таких, что $i, j^{\prime} \in \operatorname{Im} \varphi$. Затем проинтегрируем $F$ по всем непереименованным переменным. Результат мы обозначаем через $(\varphi F)\left(x_{1}, \ldots, x_{n}\right)$.

В дальнейшем нам будет удобно для обозначения разбиений использовать координаты Фробениуса (см. [7]). Мы будем записывать разбиение $\lambda$ в следующем виде: $\lambda=\left(p_{1}, \ldots, p_{d} \mid q_{1}, \ldots, q_{d}\right)$, где $p_{i}=\lambda_{i}-i$ - это число клеток в $i$-й строке диаграммы Юнга, соответствующей $\lambda$, справа от диагонали, $q_{i}=\lambda_{i}^{\prime}-i$ - число клеток в $i$-м столбце ниже диагонали, a $d$ - количество диагональных клеток ( $\lambda^{\prime}$ обозначает транспонированную диаграмму). Как правило, мы не различаем разбиения и соответствующие диаграммы Юнга.

ТЕОРема 2.1. Пусть $P$ - веролтностнал мера на $\widetilde{\Omega}$, удовлетворяюшал условиям (1.2), (1.4) и (1.5). Предположим, ито для каждого $d=1,2, \ldots$ сушествует обобшенная функиия $I_{d}\left(r_{1}, s_{1} ; \ldots ; r_{d}, s_{d}\right) \in \mathscr{V}_{2 d}$, такая, что

(1) $I_{d}$ кососимметрична относительно перестановок множества $\left(r_{1}, \ldots, r_{d}\right)$ u перестановок множества $\left(s_{1}, \ldots, s_{d}\right)$;

(2) $\operatorname{supp} I_{d} \subset \mathbb{R}_{+}^{2 d}$;

(3) моменты $I_{d}$ имеют вид

$$
\left\langle I_{d}, r_{1}^{p_{1}} s_{1}^{q_{1}} \cdots r_{d}^{p_{d}} s_{d}^{q_{d}}\right\rangle=\int_{\widetilde{\Omega}} \tilde{s}_{\left(p_{1}, \ldots, p_{d} \mid q_{1}, \ldots, q_{d}\right)}(\omega) P(d \omega) .
$$

Тогда на множестве

$$
\left(\mathbb{R}^{*}\right)^{n} \cap\left\{\left(x_{1}, \ldots, x_{n}\right) \mid \prod_{i<j}\left(x_{i}-x_{j}\right) \neq 0\right\}
$$

корреляиионнье функиии прочесса ЯР имеют вид

$2 \partial e$

$$
\rho_{n}\left(x_{1} \ldots, x_{n}\right)=\sum_{d \geqslant n / 2}^{n} \frac{1}{d !} \sum_{\varphi \in \Phi_{n, d}}\left(\varphi H_{d}\right)\left(x_{1}, \ldots, x_{n}\right)
$$

$$
H_{d}\left(r_{1}, s_{1} ; \ldots ; r_{d}, s_{d}\right)=\frac{I_{d}\left(r_{1},-s_{1} ; \ldots ; r_{d},-s_{d}\right)}{\prod_{i=1}^{d}\left(r_{i}+\left|s_{i}\right|\right)} .
$$

Необходимое и достаточное условие для того, чтобы диагонали $\left\{x_{i}=x_{j}\right\}$ были множествами меры нуль относительно корреляционных мер, будет приведено в $§ 10$.

\section{§3. Метод}

Мы будем следовать подходу, предложенному Г. И. Ольшанским в [9]. Краткое описание этого подхода приводится ниже.

Определим меру $\sigma_{n}(d x)$ на $\mathbb{R}^{n}$ следующим образом:

$$
\sigma_{n}=\int_{\widetilde{\Omega}}\left(\sum_{i=1}^{\infty} \alpha_{i} M_{\alpha_{i}}+\sum_{i=1}^{\infty} \beta_{i} M_{-\beta_{i}}+\left(\tau-\sum_{i=1}^{\infty}\left(\alpha_{i}+\beta_{i}\right)\right) M_{0}\right)^{\otimes n} P(d \omega),
$$


где $M_{x}$ обозначает дельта-меру в точке $x$.

Три утверждения ниже объясняют наш интерес к мерам $\left\{\sigma_{n}\right\}$

ПРЕДЛОЖЕНИЕ 3.1 (Ольшанский [9]). Для всякого $n=1,2, \ldots$ на множестве $\left(\mathbb{R}^{*}\right)^{n} \cap\left\{\left(x_{1}, \ldots, x_{n}\right) \mid \prod_{i<j}\left(x_{i}-x_{j}\right) \neq 0\right\}$ имеет место равенство

$$
\rho_{n}\left(d x_{1}, \ldots, d x_{n}\right)=\frac{1}{\left|x_{1} \cdots x_{n}\right|} \sigma_{n}\left(d x_{1}, \ldots, d x_{n}\right) .
$$

ПРЕДЛОЖЕНИЕ 3.2 (Ольшанский $[9,2]$ ). Все моменты меры $\sigma_{n}$ конечны; их связь с интегралами (1.3) дается формулой

$$
\int_{\mathbb{R}^{n}} x_{1}^{l_{1}} \cdots x_{n}^{l_{n}} \sigma_{n}\left(d x_{1}, \ldots, d x_{n}\right)=\sum_{\lambda,|\lambda|=\sum_{i}\left(l_{i}+1\right)} \chi_{\left(l_{1}+1, \ldots, l_{n}+1\right)}^{\lambda} \phi(\lambda) .
$$

ПРЕДЛОЖЕНИЕ 3.3. Если выполнено условие (1.5), то $\sigma_{n} \in \mathscr{V}_{n}$ для всех $n=1,2, \ldots$.

ДокАЗАТЕЛЬСТво. Для любого положительного $b<a / n(a$ из (1.5))

$$
\int_{\mathbb{R}^{n}} \sigma_{n}(x) e^{b\left(\left|x_{1}\right|+\cdots+\left|x_{n}\right|\right)} d x \leqslant \int_{\widetilde{\Omega}} \tau^{n}(\omega) e^{n b \cdot \tau(\omega)} P(d \omega)<\infty .
$$

Поскольку $\sqrt{x_{1}^{2}+\cdots+x_{n}^{2}} \leqslant\left|x_{1}\right|+\cdots+\left|x_{n}\right|$, мы получаем, что $E_{n}(b x) \cdot \sigma_{n}(x) \in$ $\mathscr{S}^{\prime}\left(\mathbb{R}^{n}\right)$.

Наша цель состоит в том, чтобы вычислить $\left\{\sigma_{n}\right\}$ с помощью (3.3) и затем извлечь корреляционные функции из (3.2). Основная техническая трудность состоит в участии в правой части формулы (3.3) характеров симметрических групп, для которых нет явных формул, а есть лишь рекуррентная вычислительная процедура, называемая правилом Мурнагана-Накаямь. Следующий параграф посвящен преобразованию этой процедуры к удобному для нас виду.

\section{$\S 4$. Правило Мурнагана-Накаямы}

Определение косого крюка читатель может найти в книге [7].

ПрЕдложЕНиЕ 4.1 (правило Мурнагана-Накаямы [7, § I.7, Example 5]). Для произвольных разбиений $\lambda$ и $\rho,|\lambda|=|\rho|$,

$$
\chi_{\rho}^{\lambda}=\sum_{S}(-1)^{\mathrm{ht}(S)},
$$

где суммирование производится по всем последовательностям разбиений $S=$ $\left(\lambda^{(0)}, \lambda^{(1)}, \ldots, \lambda^{(m)}\right)$, maкuм, ито $m=l(\rho), 0=\lambda^{(0)} \subset \lambda^{(1)} \subset \cdots \subset \lambda^{(m)} \subset \lambda u$ каждая разность $\lambda^{(i)}-\lambda^{(i-1)}$ является косыл $\rho_{i}$-крюком, $а$

$$
\operatorname{ht}(S)=\sum_{i} \operatorname{ht}\left(\lambda^{(i)}-\lambda^{(i-1)}\right)
$$

(высота косого крюка (обозначаемая $\mathrm{ht}(\cdot))$ есть уменьшенное на единичу число строк, которое он занимает).

Наши дальнейшие рассуждения основаны на следующей простой лемме.

ЛЕмма 4.2. Удаление косого $\theta$-крюка из диаграммь Юнга $\lambda$ с координатами Фробениуса $\left(p_{1}, \ldots, p_{d} \mid q_{1}, \ldots, q_{d}\right)$ равносильно одной из трех следуюших операций: 
(1) если крюк лежит выце диагонали и занимает строки $c i$-й по $j$-ю, то $i$-я р-координата Фробениуса уменьшается на $\theta$ и ставится на $j$-е место:

$$
\left(p_{1}, \ldots, p_{d}\right) \longrightarrow\left(p_{1}, \ldots, p_{i-1}, p_{i+1}, \ldots, p_{j}, p_{i}-\theta, p_{j+1}, \ldots, p_{d}\right),
$$

u вилсота крюка равна $j-i$;

(2) если крюк лежит ниже диагонали и занимает столбць $c ~ i$-го по $j$-й, то $i$-я q-координата Фробениуса уменьшается на $\theta$ и ставится на $j$-е место:

$$
\left(q_{1}, \ldots, q_{d}\right) \longrightarrow\left(q_{1}, \ldots, q_{i-1}, q_{i+1}, \ldots, q_{j}, q_{i}-\theta, q_{j+1}, \ldots, q_{d}\right),
$$

u выссота крюка равна $\theta-1+i-j$;

(3) если крюк пересекает диагональ и занимает строки, начиная $c ~ i-u, ~ u$ столбць, начиная с $j$-го, то $i$-я $p$-координата и $j$-я q-координата удаляютсл из набора координат Фробениуса; при этом $p_{i}+q_{j}=\theta-1$ и высота крюка равна $q_{j}+j-i$.

Это утверждение доказывается прямой проверкой. При вычислении высоты крюков полезно учитывать, что мощность косого крюка равна сумме количества занимаемых им строк и количества занимаемых им столбцов минус единица.

Таким образом, всякое исчерпывание диаграммы Юнга косыми крюками индуцирует исчерпывание координат Фробениуса операциями (1), (2), (3) леммы 4.2. А такое исчерпывание разбивает координаты Фробениуса на пары - в одну пару попадают $p$ - и $q$-координаты, участвующие в одной операции типа (3). Кроме того, перед тем, как попасть в (3), каждая $p$-координата (и q-координата) может подпадать под операцию (1) (соответственно (2)). Тем самым исчерпывание косыми крюками доставляет некоторую последовательность действий с координатами Фробениуса, которую мы будем называть структурой. Одна и та же структура может отвечать разным исчерпываниям одной и той же диаграммы и даже исчерпываниям разных диаграмм.

Нашей ближайшей задачей является переписывание правой части формулы (4.1) как суммы по всевозможным структурам некоторых выражений. Затем мы подставим полученную формулу в (3.3) и разобьем проблему моментов для $\sigma_{n}$ на набор более простых задач, которые будут параметризоваться различными структурами. Каждая из получившихся более простых проблем моментов будет после этого явно решена.

Для формализации сказанного выше нам понадобятся некоторые обозначения.

Мы будем рассматривать некоторые переменные, которые принимают целые неотрицательные значения. Будем называть эти переменные линейныли горизонтальными и линейными вертикальньлми блоками.

Мы также будем рассматривать переменные, возможными значениями которых являются пары $(p, q) \in\{0,1, \ldots\}^{2}$. Такие переменные будут называться крюковыми блоками.

Числом заполнения линейного блока называется его значение. Для крюкового блока будем различать $p$-число заполнения и q-число заполнения.

Мошностью линейного блока назовем его значение, а мощностью крюкового блока - число $p+q+1$.

Определим фрагмент как частично упорядоченное конечное множество блоков, удовлетворяющее следующим условиям: 
(i) есть ровно один крюковой блок, который предшествует всем остальным;

(ii) все линейные горизонтальные блоки линейно упорядочены;

(iii) все линейные вертикальные блоки линейно упорядочены;

(iv) других отношений порядка нет.

Наконец, назовем структурой конечное множество фрагментов со сквозной нумерацией всех блоков всех фрагментов, причем порядок, задаваемый этой нумерацией, должен быть согласован с внутренними частичными порядками фрагментов.

Структура называется заполненной, если известны числа заполнения всех ее блоков.

Мощностью структуры называется упорядоченный (в соответствии с нумерацией блоков) набор мощностей ее блоков.

Будем обозначать через $v(T)$ количество вертикальных блоков структуры $T$.

Определим $p$-число ( $q$-число) заполнения фрагмента как сумму $p$-числа заполнения крюкового блока и чисел заполнения линейных горизонтальных блоков этого фрагмента ( $q$-числа заполнения крюкового блока и чисел заполнения линейных вертикальных блоков соответственно).

Таким образом, заполненная структура $T$ с $d$ фрагментами доставляет набор пар

$$
\left\{\left(P_{1}, Q_{1}\right), \ldots,\left(P_{d}, Q_{d}\right)\right\}
$$

$p$ - и $q$-чисел заполнения фрагментов.

Определим знак структуры $T$ как

$$
\operatorname{sgn} T=\operatorname{sgn}\left(\prod_{i<j}\left(P_{i}-P_{j}\right)\left(Q_{i}-Q_{j}\right)\right)(-1)^{\sum Q_{j}+v(T)} .
$$

ПРЕДЛОЖЕНИЕ 4.3. Для произвольных разбиений $\lambda$ u $\rho,|\lambda|=|\rho|$,

$$
\chi_{\rho}^{\lambda}=\sum_{T} \operatorname{sgn} T,
$$

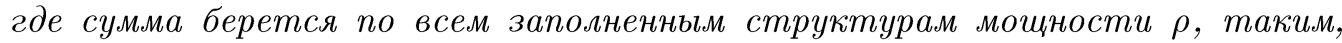
что наборы $\left\{P_{1}, \ldots, P_{d}\right\}$ u $\left\{Q_{1}, \ldots, Q_{d}\right\} p$ - и q-чисел заполнения структуры $T$ совпадают с точностью до порядка с наборами $p$ - и q-координат Фробениуса разбиения $\lambda$.

ДокАЗАТЕЛЬство. Назовем структуры, полученные из данной отбрасыванием нескольких последних (согласно нумерации) блоков, подструктурами данной структуры. Заполнение структуры, очевидно, индуцирует заполнение всех ее подструктур. Мы скажем, что заполненная структура является правильной, если знаки всех ее подструктур (включая ее саму) отличны от нуля. Это равносильно тому, что $p$ - и $q$-числа заполнения фрагментов каждой подструктуры попарно различны.

Утверждение предложения 4.3 немедленно вытекает из следующих лемм.

Лемма 4.4. Правильные структуры, участвуюшие в сумме (4.3), находятся во взаимно однозначном соответствии с последовательностями $S$ из предложения 4.1. При этом

$$
\operatorname{sgn} T=(-1)^{\mathrm{ht}(S)}
$$

для соответствующих $T$ и $S$. 
ЛЕмма 4.5. Вклад неправильных структур в сумму (4.3) равен нулю.

ДокАЗАТЕЛЬСтво ЛЕммы 4.4. Взаимно однозначное соответствие между рассматриваемыми множествами определяется таким образом, что операции (1), (2), (3) леммы 4.2 соответствуют удалению из структуры линейного горизонтального, линейного вертикального и крюкового блоков соответствующей мощности. Формально можно построить требуемое соответствие индукцией по числу $k$ блоков в структуре (равному длине разбиения $\rho$ ). При $k=1$ последовательность $S$ вида $\varnothing=\lambda^{(0)} \subset \lambda^{(1)}=\lambda=(p \mid q)$ отвечает структуре $T$ с одним крюковым блоком $(p, q)$. При этом знаки $(-1)^{\mathrm{ht}(S)}$ и $\operatorname{sgn} T$, очевидно, совпадают и равны $(-1)^{q}$. Далее, при добавлении к последовательности $S$ длины $k$ косого крюка $\lambda^{(k+1)}-\lambda^{(k)}$ мы добавляем к соответствующей структуре $T$ новый блок мощности $\left|\lambda^{(k+1)}-\lambda^{(k)}\right|$. Возможны три варианта: крюк лежит над диагональю, под диагональю или пересекает диагональ.

Рассмотрим первый вариант. Согласно лемме 4.2, одна из $p$-координат Фробениуса диаграммы $\lambda^{(k)}$ увеличилась на $\left|\lambda^{(k+1)}-\lambda^{(k)}\right|$. Мы находим в структуpe $T$ фрагмент с $p$-числом заполнения, равным этой изменяющейся координате Фробениуса, и добавляем в него новый линейный горизонтальный блок мощности $\left|\lambda^{(k+1)}-\lambda^{(k)}\right|$. При этом изменение $\operatorname{sgn} T$ равно изменению знака произведения $\prod_{i<j}\left(P_{i}-P_{j}\right)$ в (4.2), т. е. если $i, j$ такие же, как в операции (1) леммы 4.2, то изменение равно $(-1)^{j-i}=(-1)^{\mathrm{ht}\left(\lambda^{(k+1)}-\lambda^{(k)}\right)}$. Таким образом, если знаки у $S$ и $T$ совпадают, то знаки у новых, только что построенных $S$ и $T$ тоже совпадают.

Второй вариант соответствует добавлению линейного вертикального блока мощности $\left|\lambda^{(k+1)}-\lambda^{(k)}\right|$, а третий — добавлению крюкового блока с $p$-числом заполнения, равным числу клеток крюка $\lambda^{(k+1)}-\lambda^{(k)}$ над диагональю, и $q$-числом заполнения, равным числу клеток крюка $\lambda^{(k+1)}-\lambda^{(k)}$ под диагональю. Тот факт, что и в этих двух случаях изменение $\operatorname{sgn} T$ равно $(-1)^{\mathrm{ht}\left(\lambda^{(k+1)}-\lambda^{(k)}\right)}$, проверяется непосредственно.

Условие правильности структур существенно, поскольку $p$ - и $q$-координаты Фробениуса диаграмм Юнга попарно различны.

ДокаЗАТЕЛЬСтво леммы 4.5. Сразу же исключим из рассмотрения структуры с равными $p$ - или $q$-числами заполнения фрагментов - их знак равен нулю. Все остальные неправильные структуры мы разобьем на пары так, что в одной паре структуры будут иметь разные знаки.

Берем неправильную структуру. Выбираем ее наибольшую подструктуру с нулевым знаком. Пусть для определенности равны $p$-числа каких-то двух фрагментов этой подструктуры. Поменяем местами в этих двух фрагментах все блоки, которые отличают исходную структуру от выбранной подструктуры. Мы получим новую структуру, которая и будет парой для исходной. Очевидно, что описанная процедура инволютивна и знаки структур в одной паре различны $\left(\prod_{i<j}\left(P_{i}-P_{j}\right)\right.$ или, в случае $q$-чисел, $\prod_{i<j}\left(Q_{i}-Q_{j}\right)$ меняет знак $)$.

Таким образом, предложение 4.3 доказано.

ЗАмЕчАНИЕ 4.6. Сумма в правой части формулы (4.3) может быть переписана как двойная - внешнее суммирование ведется по всем структурам с $l(\rho)$ блоками, а внутреннее - по всем заполнениям данной структуры, таким, что выполнены условия предложения 4.3. 


\section{§5. Упрощение проблемы моментов}

Зафиксируем произвольную структуру $T$. Обозначим через $n$ число ее блоков и через $d$ число фрагментов. Занумеруем фрагменты произвольным образом числами от 1 до $d$. Обозначим количество линейных горизонтальных блоков в $i$-м фрагменте через $\mu_{i}$, а количество линейных вертикальных блоков через $\nu_{i}$. Тогда

$$
\sum_{i=1}^{d}\left(\mu_{i}+\nu_{i}+1\right)=n
$$

(в каждом фрагменте есть ровно один крюковой блок). В этих обозначениях число вертикальных блоков $v(T)$ равно $\sum_{i=1}^{d} \nu_{i}$.

Поставим в соответствие каждому из $n$ блоков структуры $T$ переменную, причем у каждой такой переменной будет два имени. Первое имя переменной, отвечающей $j$-му блоку, - это $x_{j}$. Набор вторых имен состоит из букв

$$
a_{i}, \quad b_{i}^{j}, j=1, \ldots, \mu_{i}, \quad c_{i}^{j}, j=1, \ldots, \nu_{i}, \quad i=1, \ldots, d ;
$$

буква $a_{i}$ соответствует крюковому блоку $i$-го фрагмента, $b_{i}^{j}, j=1, \ldots, \mu_{i}$, соответствуют горизонтальным блокам $i$-го фрагмента и $c_{i}^{j}, j=1, \ldots, \nu_{i}$, вертикальным блокам $i$-го фрагмента

Аналогично этому переобозначим числа $\left(l_{1}, \ldots, l_{n}\right.$ ) (участвующие в $(3.3)$ ) буквами $A_{i}, B_{i}^{j}, C_{i}^{j}$.

Мы можем рассматривать функцию $\phi$, определенную в (1.3), как функцию на парах $\left(p_{1}, \ldots, p_{d} \mid q_{1}, \ldots, q_{d}\right)$ наборов строго убывающих натуральных чисел (координатах Фробениуса диаграмм Юнга). Продолжим эту функцию на множество пар произвольных наборов натуральных чисел с одинаковым числом элементов так, чтобы $\phi\left(p_{1}, \ldots, p_{d} \mid q_{1}, \ldots, q_{d}\right)$ была кососимметричной относительно перестановок множества $\left\{p_{i}\right\}_{i=1}^{d}$ и перестановок множества $\left\{q_{i}\right\}_{i=1}^{d}$. Ясно, что такое продолжение единственно.

ПРЕДЛОЖЕНИЕ 5.1. Пусть для каждой структурь $T$ с $n$ блоками существует распределение

$$
\sigma_{n}^{T}\left(\left\{a_{i}\right\}_{i=1}^{d},\left\{b_{i}^{j}\right\}_{i=1, j=1}^{d, \mu_{i}},\left\{c_{i}^{j}\right\}_{i=1, j=1}^{d, \nu_{i}}\right) \in \mathscr{V}_{n}
$$

(пространство $\mathscr{V}_{n}$ было определено в конце §1) с моментами

$$
\begin{aligned}
& \left\langle\sigma_{n}^{T}, \prod_{i=1}^{d}\left(a_{i}^{A_{i}}\left(b_{i}^{1}\right)^{B_{i}^{1}} \cdots\left(b_{i}^{\mu_{i}}\right)^{B_{i}^{\mu_{i}}}\left(c_{i}^{1}\right)^{C_{i}^{1}} \cdots\left(c_{i}^{\nu_{i}}\right)^{C_{i}^{\nu_{i}}}\right)\right\rangle \\
& \quad=\sum_{\substack{A_{1}^{\prime}, A_{1}^{\prime \prime} \geqslant 0, A_{1}^{\prime}+A_{1}^{\prime \prime}=A_{1}}} \cdots \sum_{\substack{A_{d}^{\prime}, A_{d}^{\prime \prime} \geqslant 0, A_{d}^{\prime}+A_{d}^{\prime \prime}=A_{d}}}(-1)^{\sum_{j} Q_{j}+v(T)} \phi\left(P_{1}, \ldots, P_{d} \mid Q_{1}, \ldots, Q_{d}\right),
\end{aligned}
$$

2дe

$$
P_{i}=A_{i}^{\prime}+\sum_{j=1}^{\mu_{i}}\left(B_{i}^{j}+1\right), \quad Q_{i}=A_{i}^{\prime \prime}+\sum_{j=1}^{\nu_{i}}\left(C_{i}^{j}+1\right)
$$


Тогда обобщенная функиия

$$
\sigma_{n}=\sum_{T} \sigma_{n}^{T}
$$

где суммирование производится по всем структурам с п блоками, доставляет решение проблемы моментов (3.3).

Прежде чем доказывать это утверждение, объясним, чего нам удалось достичь. Нам удалось разбить проблему моментов (3.3) на конечное число задач (5.1), причем если считать функцию $\phi$ известной, то задачи (5.1) значительно проще, чем (3.3): формула (5.1) не содержит характеров $\chi^{\lambda}$ и суммирование в ней ведется по целым числам от 0 до $A_{i}, i=1, \ldots, d$, что, конечно, лучше, чем суммирование по диаграммам Юнга в (3.3).

ДОКАЗАТЕЛЬСТВо ПРЕДЛОЖЕНИЯ 5.1. Подставим формулу (4.3) в правую часть формулы (3.3) и воспользуемся замечанием 4.6. Имеем

$$
\left\langle\sigma_{n}, x_{1}^{l_{1}} \cdots x_{n}^{l_{n}}\right\rangle=\sum_{T} \sum_{\text {заполнения } T} \operatorname{sgn} T \cdot \phi(\lambda) .
$$

Зафиксируем структуру $T$ с $n$ блоками. Свобода в заполнении структуры с известными мощностями блоков состоит лишь в выборе $p$ - и $q$-чисел заполнения крюковых блоков, причем сумма $p$ - и $q$-чисел в каждом крюковом блоке фиксирована (и равна мощности блока минус единица).

В нашем случае имеется $d$ крюковых блоков мощности $A_{i}, i=1, \ldots, d$. Обозначим их $p$ - и $q$-числа заполнения через $A_{i}^{\prime}, A_{i}^{\prime \prime}, i=1, \ldots, d$. Тогда суммирование по всевозможным заполнениям структуры $T$ данной мощности обратится в суммирование по парам $\left(A_{i}^{\prime}, A_{i}^{\prime \prime}\right)$, таким, что $A_{i}^{\prime}+A_{i}^{\prime \prime}=A_{i}$. Далее, $P_{i}$ и $Q_{i}$ из $(5.2)$ - это $p$ - и $q$-числа заполнения $i$-го фрагмента. Поскольку $\phi$ кососимметрична, мы имеем

$$
\operatorname{sgn}\left(\prod_{i<j}\left(P_{i}-P_{j}\right)\left(Q_{i}-Q_{j}\right)\right) \phi(\lambda)=\phi\left(P_{1}, \ldots, P_{d} \mid Q_{1}, \ldots, Q_{d}\right) .
$$

Знак $(-1)^{\sum_{j} Q_{j}+v(T)}$ в $(5.1)$ - это отношение $\operatorname{sgn} T$ и знака левой части формулы (5.5).

Наша следующая задача - выражение обобщенных функций $\sigma_{n}^{T}$ через функции $I_{d}, d=1,2, \ldots$, введенные в теореме 2.1. Следующий параграф снабдит нас необходимыми для этого средствами.

\section{§6. Три операции над обобщенными функциями}

ПРЕДЛОЖЕНИЕ 6.1 (умножение на моном). Для всякой обобщенной функции $f\left(\xi_{1}, \ldots, \xi_{k}\right) \in \mathscr{V}_{k}, k \geqslant 1$, с моментами

$$
\left\langle f\left(\xi_{1}, \ldots, \xi_{k}\right), \xi_{1}^{i_{1}} \cdots \xi_{k}^{i_{k}}\right\rangle=m_{i_{1}, \ldots, i_{k}}
$$

и любого набора неотричательных чельх иисел $j_{1}, \ldots, j_{k}$ обобщеннал функиия $\xi_{1}^{j_{1}} \cdots \xi_{k}^{j_{k}} f\left(\xi_{1}, \ldots, \xi_{k}\right)$ тажже принадлежит $\mathscr{V}_{k} u$

$$
\left\langle\xi_{1}^{j_{1}} \cdots \xi_{k}^{j_{k}} f\left(\xi_{1}, \ldots, \xi_{k}\right), \xi_{1}^{i_{1}} \cdots \xi_{k}^{i_{k}}\right\rangle=m_{i_{1}+j_{1}, \ldots, i_{k}+j_{k}} .
$$

Доказательство очевидно. 
ПРЕДЛОЖЕНИЕ 6.2 («свертКа»). Для всякой обобиенной функиии $f\left(\xi_{1}, \xi_{2}, \eta_{1}\right.$, $\left.\ldots, \eta_{k}\right) \in \mathscr{V}_{k+2}, k \geqslant 0$, смоментами

$$
\left\langle f\left(\xi_{1}, \xi_{2}, \eta_{1}, \ldots, \eta_{k}\right), \xi_{1}^{i_{1}} \xi_{2}^{i_{2}} \eta_{1}^{j_{1}} \cdots \eta_{k}^{j_{k}}\right\rangle=m_{i_{1}, i_{2}, j_{1}, \ldots, j_{k}}
$$

существует обобщенная функиия $g\left(\xi, \eta_{1}, \ldots, \eta_{k}\right) \in \mathscr{V}_{k+1}$ с моментами

$$
\left\langle g\left(\xi, \eta_{1}, \ldots, \eta_{k}\right), \xi^{i} \eta_{1}^{j_{1}} \ldots \eta_{k}^{j_{k}}\right\rangle=\sum_{\substack{i_{1}, i_{2} \geqslant 0 \\ i_{1}+i_{2}=i}}(-1)^{i_{2}} m_{i_{1}, i_{2}, j_{1}, \ldots, j_{k}} .
$$

Если, кроме того, supp $f \subset\left\{\xi_{1} \geqslant 0, \xi_{2} \geqslant 0\right\}$, то в области $\{\xi \neq 0\}$

$$
g\left(\xi, \eta_{1}, \ldots, \eta_{k}\right)=\int_{y \geqslant 0} \frac{|\xi|}{|\xi|+y}\left(f\left(\xi, y, \eta_{1}, \ldots, \eta_{k}\right)+f\left(y,-\xi, \eta_{1}, \ldots, \eta_{k}\right)\right) d y
$$

Для функции $g$ мы будем использовать обозначение

$$
g\left(\xi, \eta_{1}, \ldots, \eta_{k}\right)=\mathscr{C}_{\xi_{1} \xi_{2}}^{\xi}\left[\left(f\left(\xi_{1}, \xi_{2}, \eta_{1}, \ldots, \eta_{k}\right)\right]\right.
$$

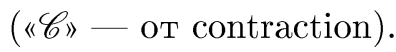

ДокАЗАтЕльство. Значение $g$ на пробной функции $\psi$ дается формулой

$$
\langle g, \psi\rangle=\left\langle f, \frac{\xi_{1} \psi\left(\xi_{1}, \eta_{1}, \ldots, \eta_{k}\right)+\xi_{2} \psi\left(-\xi_{2}, \eta_{1}, \ldots, \eta_{k}\right)}{\xi_{1}+\xi_{2}}\right\rangle .
$$

ПРЕДЛОЖЕНИЕ 6.3 («диагонализация»). Для всякой обобиенной функиии $f\left(\xi, \eta_{1}, \ldots, \eta_{k}\right) \in \mathscr{V}_{k+1}, k \geqslant 0$, с моментами

$$
\left\langle f\left(\xi, \eta_{1}, \ldots, \eta_{k}\right), \xi^{i} \eta_{1}^{j_{1}} \cdots \eta_{k}^{j_{k}}\right\rangle=m_{i, j_{1}, \ldots, j_{k}}
$$

обобшенная функиия

$$
g\left(\xi_{1}, \xi_{2}, \eta_{1}, \ldots, \eta_{k}\right)=f\left(\xi_{1}, \eta_{1}, \ldots, \eta_{k}\right) \delta\left(\xi_{2}-\xi_{1}\right)
$$

принадлежит $\mathscr{V}_{k+2}$. Ее моменты равнь

$$
\left\langle g\left(\xi_{1}, \xi_{2}, \eta_{1}, \ldots, \eta_{k}\right), \xi_{1}^{i_{1}} \xi_{2}^{i_{2}} \eta_{1}^{j_{1}} \cdots \eta_{k}^{j_{k}}\right\rangle=m_{i_{1}+i_{2}, j_{1}, \ldots, j_{k}} .
$$

Мы будем использовать обозначение

$$
g\left(\xi_{1}, \xi_{2}, \eta_{1}, \ldots, \eta_{k}\right)=\mathscr{D}_{\xi}^{\xi_{1} \xi_{2}}\left[f\left(\xi, \eta_{1}, \ldots, \eta_{k}\right)\right]
$$

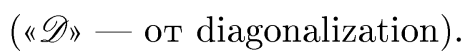

Доказательство очевидно.

\section{$\S 7$. Решение упрощенной проблемы моментов}

Начиная с этого параграфа, мы постулируем существование обобщенных функций $I_{d}\left(r_{1}, s_{1} ; \ldots ; r_{d}, s_{d}\right) \in \mathscr{N}_{2 d}, d=1,2, \ldots$, удовлетворяющих условиям теоремы 2.1.

Зафиксируем произвольную структуру $T$. Мы будем пользоваться обозначениями $§ 5$.

Для каждого $i=1, \ldots, d$ положим

$$
\mathscr{C}_{i}=\mathscr{C}_{a_{i}^{\prime}, a_{i}^{\prime \prime}}^{a_{i}}, \quad \mathscr{D}_{i}^{\prime}=\prod_{j=1}^{\mu_{i}} \mathscr{D}_{a_{i}^{\prime}}^{a_{i}^{\prime}, b_{i}^{j}}, \quad \mathscr{D}_{i}^{\prime \prime}=\prod_{j=1}^{\nu_{i}} \mathscr{D}_{a_{i}^{\prime \prime}}^{a_{i}^{\prime \prime},-c_{i}^{j}}, \quad \mathscr{D}_{i}=\mathscr{D}_{i}^{\prime} \mathscr{D}_{i}^{\prime \prime} .
$$

Обозначим также через $\mathscr{M}_{i}, i=1, \ldots, d$, оператор умножения на $\left(a_{i}^{\prime}\right)^{\mu_{i}}\left(a_{i}^{\prime \prime}\right)^{\nu_{i}}$. 
ПРЕДЛОЖЕНИЕ 7.1. Обобщенная функиия

$$
\sigma_{n}^{T}=\left(\prod_{i=1}^{d} \mathscr{C}_{i}\right)\left(\prod_{i=1}^{d} \mathscr{D}_{i}\right)\left(\prod_{i=1}^{d} \mathscr{M}_{i}\right) \cdot I_{d}\left(a_{1}^{\prime}, a_{1}^{\prime \prime} ; \ldots ; a_{d}^{\prime}, a_{d}^{\prime \prime}\right)
$$

принадлежит пространству $\mathscr{V}_{n}$ и доставляет решение проблемь моментов (5.1).

ДокАЗАТЕльство. Мы проведем доказательство для случая $d=1$, в общем случае рассуждения совершенно аналогичны. Мы будем опускать нижний индекс у всех букв (кроме $I_{1}$ ), поскольку он всегда будет равен 1 .

Из условий (1), (3) теоремы 2.1 следует (мы используем обозначение (1.3)), чTо

$$
\left\langle I_{1}\left(a^{\prime}, a^{\prime \prime}\right),\left(a^{\prime}\right)^{A^{\prime}}\left(a^{\prime \prime}\right)^{A^{\prime \prime}}\right\rangle=\phi\left(\left(A^{\prime} \mid A^{\prime \prime}\right)\right) .
$$

Предложение 6.1 дает

$$
\left\langle\left(\mathscr{M} I_{1}\right)\left(a^{\prime}, a^{\prime \prime}\right),\left(a^{\prime}\right)^{A^{\prime}}\left(a^{\prime \prime}\right)^{A^{\prime \prime}}\right\rangle=\phi\left(\left(A^{\prime}+\mu \mid A^{\prime \prime}+\nu\right)\right) .
$$

Далее из предложения 6.3 мы получаем

$$
\begin{gathered}
\left\langle\left(\mathscr{D} \mathscr{M} I_{1}\right)\left(a^{\prime}, a^{\prime \prime},\left\{b^{j}\right\}_{j=1}^{\mu},\left\{c^{j}\right\}_{j=1}^{\nu}\right),\left(a^{\prime}\right)^{A^{\prime}}\left(a^{\prime \prime}\right)^{A^{\prime \prime}} \prod_{j=1}^{\mu}\left(b^{j}\right)^{B^{j}} \prod_{j=1}^{\nu}\left(c^{j}\right)^{C^{j}}\right\rangle \\
=(-1)^{\sum_{j=1}^{\nu} C^{j}} \phi((P \mid Q)),
\end{gathered}
$$

где (cp. (5.2)),

$$
P=A^{\prime}+\sum_{j=1}^{\mu}\left(B^{j}+1\right), \quad Q=A^{\prime \prime}+\sum_{j=1}^{\nu}\left(C^{j}+1\right) .
$$

Знак $(-1)^{\sum_{j=1}^{\nu} C^{j}}$ появился из-за того, что для одномерного распределения $g(\eta)$

$$
\left\langle g(\eta), \eta^{l}\right\rangle=(-1)^{l}\left\langle g(-\eta), \eta^{l}\right\rangle .
$$

В нашем случае мы применяем этот факт к переменным $c^{j}$, которые входят в определение $\mathscr{D}^{\prime \prime}$ со знаком минус.

Наконец, применяя предложение 6.2, мы получаем

$$
\begin{aligned}
& \left\langle\left(\mathscr{C} \mathscr{D} \mathscr{M} I_{1}\right)\left(a^{\prime}, a^{\prime \prime},\left\{b^{j}\right\}_{j=1}^{\mu},\left\{c^{j}\right\}_{j=1}^{\nu}\right), a^{A} \prod_{j=1}^{\mu}\left(b^{j}\right)^{B^{j}} \prod_{j=1}^{\nu}\left(c^{j}\right)^{C^{j}}\right\rangle \\
& =\sum_{\substack{A^{\prime}, A^{\prime \prime} \geqslant 0 \\
A^{\prime}+A^{\prime \prime}=A}}(-1)^{A^{\prime \prime}+\sum_{j=1}^{\nu} C^{j}} \phi((P \mid Q)),
\end{aligned}
$$

где $P$ и $Q$ определены в (7.2). Остается заметить, что

$$
(-1)^{A^{\prime \prime}+\sum_{i=1}^{\nu} C^{j}}=(-1)^{Q-\nu}=(-1)^{Q+\nu}=(-1)^{Q+v(T)},
$$

и (5.1) доказано.

В случае $d>1$ все те же рассуждения применяются к каждому из $d$ фрагментов. 


\section{§8. Существенные структуры}

Согласно предложению 3.1 , корреляционные функции $\rho_{n}$ восстанавливаются по $\sigma_{n}$ только на множестве, где $x_{i} \neq x_{j}$ для всех пар $i \neq j$; поэтому мы сразу можем отбросить те структуры $T$, для которых носитель обобщенной функции $\sigma_{n}^{T}$ лежит в диагональном множестве $\left\{x \mid \prod_{i<j}\left(x_{i}-x_{j}\right)=0\right\}$.

Назовем структуру существенной, если в каждом ее фрагменте имеется не более двух блоков.

ПРЕДЛОЖЕНИЕ 8.1. Если структура Т не является существенной, то

$$
\operatorname{supp} \sigma_{n}^{T} \subset\left\{\left(x_{1}, \ldots, x_{n}\right) \mid \prod_{i} x_{i} \cdot \prod_{i<j}\left(x_{i}-x_{j}\right)=0\right\} .
$$

ДокАЗАТЕльство. Мы будем использовать обозначения $\S 5$. Предположим, как при доказательстве предложения 7.1, что $T$ имеет лишь один фрагмент. Мы опять будем опускать всегда равный единице нижний индекс.

Если структура $T$ не является существенной, то возможны три случая: $\mu \geqslant 2$, $\nu \geqslant 2, \mu=\nu=1$. Если $\mu \geqslant 2$, то моменты (5.1) зависят от показателей $B^{1}, \ldots, B^{\mu}$ только через их сумму. Следовательно, $\operatorname{supp} \sigma_{n}^{T} \subset\left\{b^{1}=\cdots=b^{\mu}\right\}$. Если $\nu \geqslant 2$, то аналогично мы получаем, что $\operatorname{supp} \sigma_{n}^{T} \subset\left\{c^{1}=\cdots=c^{\nu}\right\}$.

Если же $\mu=\nu=1$, то мы применяем предложение 7.1. Имеем

$$
\begin{aligned}
\left(\mathscr{M} I_{1}\right)\left(a^{\prime}, a^{\prime \prime}\right) & =a^{\prime} a^{\prime \prime} I_{1}\left(a^{\prime}, a^{\prime \prime}\right), \\
\left(\mathscr{D} \mathscr{M} I_{1}\right)\left(a^{\prime}, a^{\prime \prime}, b, c\right) & =a^{\prime} a^{\prime \prime} I_{1}\left(a^{\prime}, a^{\prime \prime}\right) \delta\left(c+a^{\prime \prime}\right) \delta\left(b-a^{\prime}\right) .
\end{aligned}
$$

Далее, из условия (2) теоремы 2.1 следует, что $\operatorname{supp}\left(\mathscr{D} \mathscr{M} I_{1}\right) \subset\left\{a^{\prime}, a^{\prime \prime} \geqslant 0\right\}$; поэтому мы можем применить (6.1). В области $\{a \neq 0\}$

$$
\begin{aligned}
\left(\mathscr{C} \mathscr{D} \mathscr{M} I_{1}\right)(a, b, c)= & \int_{y \geqslant 0} \frac{|a|}{|a|+y}\left(a y I_{1}(a, y) \delta(c+y) \delta(b-a)\right. \\
& \left.-a y I_{1}(y,-a) \delta(c-a) \delta(b-y)\right) d y \\
& =-\frac{a^{2} c}{a-c} I_{1}(a,-c) \delta(b-a)+\frac{a^{2} b}{b-a} I_{1}(b,-a) \delta(c-a) .
\end{aligned}
$$

Очевидно, что носитель последнего выражения лежит в области $\{(a, b, c) \mid$ $(b-a)(c-a)=0\}$, а это и требовалось доказать.

В случае произвольного числа фрагментов мы пользуемся тем, что операции $\mathscr{C}_{i}, \mathscr{D}_{i}, \mathscr{M}_{i}$ с разными индексами действуют на непересекающихся наборах переменных. Поэтому применение приведенных выше рассуждений к фрагменту с более чем двумя блоками доказывает утверждение.

Таким образом, мы показали, что лишь существенные структуры дают существенный для корреляционных функций вклад в правую часть формулы (5.3).

\section{§. Доказательство основного результата}

Мы начинаем с вычисления $\sigma_{n}^{T}$ для всех сушественных структур $T$ с одним фрагментом. Таких структур три - в $T$ может быть только крюковой блок, может быть линейный горизонтальный блок вместе с крюковым и может быть линейный вертикальный блок вместе с крюковым. В наших обозначениях это соответствует значениям $\mu=\nu=0, \mu=1, \nu=0$ и $\mu=0, \nu=1$ соответственно. Обозначим эти три структуры через $T_{1}, T_{2}$ и $T_{3}$. 
ЛЕмма 9.1. В области $\{a \neq 0\}$

$$
\begin{gathered}
\sigma_{1}^{T_{1}}(a)=|a|\left(\int_{y \geqslant 0} \frac{I_{1}(a, y)}{a+y} d y+\int_{y \geqslant 0} \frac{I_{1}(y,-a)}{y+|a|} d y\right), \\
\sigma_{1}^{T_{2}}(a, b)=|a b|\left(\frac{I_{1}(b,-a)}{b+|a|}+\int_{y \geqslant 0} \frac{I_{1}(a, y)}{a+y} d y \cdot \delta(b-a)\right), \\
\sigma_{1}^{T_{3}}(a, c)=|a c|\left(\frac{I_{1}(a,-c)}{a+|c|}+\int_{y \geqslant 0} \frac{I_{1}(y,-a)}{y+|a|} d y \cdot \delta(c-a)\right) .
\end{gathered}
$$

Доказательство леммы 9.1 получается прямым применением предложения 7.1 с использованием формулы (6.1). Заметим, что носители вторых слагаемых в (9.2) и (9.3) лежат в диагональном множестве; поэтому при вычислении корреляционных функций мы можем эти слагаемые отбросить.

При вычислении $\sigma_{n}^{T}$ для произвольной существенной структуры $T$ с $n$ блоками и $d$ фрагментами для каждого из фрагментов возникает одна из трех ситуаций, моделируемых структурами $T_{1}, T_{2}, T_{3}$.

Начинаем с формулы (7.1). Как уже отмечалось в конце $\S 8$, операции $\mathscr{C}_{i}, \mathscr{D}_{i}$, $\mathscr{M}_{i}$ с разными индексами действуют на разных наборах переменных; поэтому разные фрагменты можно рассматривать независимо.

Рассмотрим фрагмент с номером $i$. Предположим, что в нем имеется единственный (крюковой) блок с номером $k$ (в сквозной нумерации блоков структуры). Тогда, применяя $\mathscr{C}_{i} \mathscr{D}_{i} \mathscr{M}_{i}$ к $I_{d}\left(a_{1}^{\prime}, a_{1}^{\prime \prime} ; \ldots ; a_{d}^{\prime}, a_{d}^{\prime \prime}\right)$, согласно $(7.1)$, мы получим два слагаемых, аналогичных стоящим в правой части формулы (9.1). Более точно, в первом слагаемом $a_{i}^{\prime}$ заменяется на $x_{k}, a_{i}^{\prime \prime}$ - на $y$, выражение делится на $x_{k}+y$ и интегрируется по $y \geqslant 0$. Во втором слагаемом мы заменяем $a_{i}^{\prime}$ на $y$, $a_{i}^{\prime \prime}$ на $-x_{k}$, делим все на $y+\left|x_{k}\right|$ и интегрируем по $y \geqslant 0$. Оба слагаемых домножаются на $\left|x_{k}\right|$.

Если $i$-й фрагмент содержит крюковой блок с номером $k$ и горизонтальный блок с номером $l$, то на внедиагональном множестве после применения $\mathscr{C}_{i} \mathscr{D}_{i} \mathscr{M}_{i}$ остается одно слагаемое, аналогичное первому слагаемому в правой части формулы (9.2): $a_{i}^{\prime}$ заменяется на $x_{l}, a_{i}^{\prime \prime}$ - на $-x_{k}$, все делится на $x_{l}+\left|x_{k}\right|$ и умножается на $\left|x_{k} x_{l}\right|$.

Наконец, если $i$-й фрагмент содержит крюковой блок с номером $k$ и вертикальный блок с номером $l$, то процедура выглядит так (ср. первое слагаемое в правой части формулы (9.3)): $a_{i}^{\prime}$ заменяется на $x_{k}, a_{i}^{\prime \prime}-$ на $-x_{l}$, все выражение делится на $x_{k}+\left|x_{l}\right|$ и умножается на $\left|x_{k} x_{l}\right|$.

Несложно увидеть, что после применения всех $\mathscr{C}_{i} \mathscr{D}_{i} \mathscr{M}_{i}$ и выбора для каждого фрагмента первого типа (т.е. с одним блоком) одного из двух слагаемых получившееся выражение будет равно, в обозначениях $\S 2$,

$$
\left|x_{1} \cdots x_{n}\right| \cdot\left(\varphi H_{d}\right)\left(x_{1}, \ldots, x_{n}\right)
$$

для некоторого $\varphi \in \Phi_{n, d}$. Более точно, для $i$-го фрагмента первого типа в случае выбора первого слагаемого

$$
\varphi(k)=i, \quad \varphi^{-1}\left(i^{\prime}\right)=\varnothing,
$$

а в случае выбора второго слагаемого

$$
\varphi(k)=i^{\prime}, \quad \varphi^{-1}(i)=\varnothing .
$$


Для $i$-го фрагмента второго типа

$$
\varphi(k)=i^{\prime}, \quad \varphi(l)=i .
$$

Для $i$-го фрагмента третьего типа

$$
\varphi(k)=i, \quad \varphi(l)=i^{\prime} .
$$

Заметим, что множитель $\left|x_{1} \cdots x_{n}\right|$ в (9.4) нам очень удобен - при переходе к корреляционным функциям (формула (3.2)) он сокрашается.

Несложно проверить, что описанная выше процедура построения $\varphi \in \Phi_{n, d}$ по структуре с $n$ блоками, $d$ упорядоченными фрагментами и выбором для каждого фрагмента с одним блоком одного из двух слагаемых является обратимой. Это почти обосновывает переход от (5.3) к (2.2). Однако изначально порядок фрагментов структур $T$, участвующих в формуле (5.3), не определен, мы произвольным образом фиксировали нумерацию фрагментов в $\S 5$ для удобства обозначений. Изменение этого порядка приводит к композиции отображения $\varphi$ с перестановкой пар $\left(i, i^{\prime}\right)$ множества $\left\{1,1^{\prime} ; \ldots ; d, d^{\prime}\right\}$. Тем самым структуры с $n$ блоками, $d$ неупорядоченными фрагментами и выбором для каждого фрагмента с одним блоком одного из двух слагаемых находятся во взаимно однозначном соответствии с орбитами симметрической группы $S_{d}$ в $\Phi_{n, d} ; S_{d}$ действует перестановками пар $\left(i, i^{\prime}\right)$, и каждая орбита содержит $d$ ! элементов. Ввиду симметричности $H_{d}\left(r_{1}, s_{1} ; \ldots ; r_{d}, s_{d}\right)$ относительно перестановок пар $\left(r_{i}, s_{i}\right)$ элементы $\varphi \in \Phi_{n, d}$ из одной орбиты дают одинаковый вклад в правую часть формулы $(2.2)$, что приводит к появлению множителя $(d !)^{-1}$. Тем самым теорема 2.1 доказана.

\section{$\S 10$. Отсутствие кратностей}

В этом параграфе мы докажем критерий того, что диагональные подмножества являются множествами меры нуль относительно корреляционных мер. Это эквивалентно тому, что с вероятностью 1 все точки случайной конфигурации различны (см. [9]). Такие процессы называются простылми. Таким образом, мы приводим необходимое и достаточное условие простоты процесса $\mathscr{P}$.

ПРЕДЛОЖЕНИЕ 10.1. Пусть выполнень условия теоремь 2.1. Вторая коррелячионная функиия в $(-\infty, 0)^{2} \sqcup(0,+\infty)^{2}$ имеет вид

$$
\begin{aligned}
\rho_{2}\left(x_{1}, x_{2}\right)= & \iint_{y_{1}, y_{2}} \frac{I_{2}\left(x_{1}, y_{1} ; x_{2}, y_{2}\right)}{\left(x_{1}+y_{1}\right)\left(x_{2}+y_{2}\right)} d y_{1} d y_{2} \\
& +\iint_{y_{1}, y_{2}} \frac{I_{2}\left(y_{1},-x_{1} ; y_{2},-x_{2}\right)}{\left(y_{1}+\left|x_{1}\right|\right)\left(y_{2}+\left|x_{2}\right|\right)} d y_{1} d y_{2} .
\end{aligned}
$$

Тогда процесс ЯР является простьим, если и только если диагональ $\left\{\left(x_{1}, x_{2}\right) \in\right.$ $\left.\left(\mathbb{R}^{*}\right)^{2} \mid x_{1}=x_{2}\right\}$ имеет меру нуль относительно второй корреляционной мерь $\rho_{2}\left(d x_{1}, d x_{2}\right)=\rho_{2}\left(x_{1}, x_{2}\right) d x_{1} d x_{2}$.

Доказательство. Вторая часть утверждения доказана в [9, Proposition 4.2]; поэтому достаточно доказать (10.1). Несложно показать, что на всем $\left(\mathbb{R}^{*}\right)^{2}$ имеет место равенство (ср. [9, Proposition 4.4])

$$
\rho_{2}\left(x_{1}, x_{2}\right)=\frac{1}{\left|x_{1} x_{2}\right|}\left\{\text { плотность }\left(\sigma_{2}\left(d x_{1}, d x_{2}\right)-\left|x_{1}\right| \sigma_{1}\left(d x_{1}\right) \delta\left(x_{1}-x_{2}\right)\right)\right\} \text {. }
$$


Существует всего одна структура с одним блоком ( $T_{1}$ из $\left.\S 9\right)$ и три структуры с двумя блоками $\left(T_{2}, T_{3}\right.$ из $\S 9$ и единственная структура с двумя крюковыми блоками, которую мы обозначим $\left.T_{4}\right)$. Таким образом (см. $\left.(5.3)\right)$,

$$
\begin{gathered}
\text { плотность }\left(\sigma_{2}\left(d x_{1}, d x_{2}\right)-\left|x_{1}\right| \sigma_{1}\left(d x_{1}\right) \delta\left(x_{1}-x_{2}\right)\right) \\
=\sum_{i=2}^{4} \sigma_{2}^{T_{i}}\left(x_{1}, x_{2}\right)-\left|x_{1}\right| \sigma_{1}^{T_{1}}\left(x_{1}\right) \delta\left(x_{1}-x_{2}\right) .
\end{gathered}
$$

Воспользуемся леммой 9.1. Легко видеть, что вторые слагаемые в формулах (9.2) и (9.3) после подстановки в правую часть формулы (10.3) сократятся с $\left|x_{1}\right| \sigma_{1}^{T_{1}}\left(x_{1}\right) \delta\left(x_{1}-x_{2}\right)$. Кроме того, носители первых слагаемых в $(9.2)$ и $(9.3)$ не пересекаются с $(-\infty, 0)^{2} \sqcup(0,+\infty)^{2}$. Следовательно, на этом множестве левая часть формулы $(10.3)$ равна $\sigma_{2}^{T_{4}}\left(x_{1}, x_{2}\right)$. Прямое вычисление распределения $\sigma_{2}^{T_{4}}$, согласно предложению 7.1 , в нужной нам области дает в точности правую часть формулы (10.1), умноженную на $\left|x_{1} x_{2}\right|$. Соотношение (10.2) завершает доказательство.

\section{ЛитерАтУРА}

1. Borodin A., Olshanski G. Point processes and the infinite symmetric group. Math. Res. Lett., 5, 799-816 (1998) (preprint version: http://xxx.lanl.gov/abs/math/ 9810015).

2. Borodin A., Olshanski G. In preparation.

3. Вершик A. М., Керов C. В. Асимптотическая теория характеров симметрической группы. Функц. анализ и его прил., 15, вып. 4, 15-27 (1981).

4. Daley D. J., Vere-Jones D. An introduction to the theory of point processes. Springer Series in Statistics, Springer-Verlag, 1988.

5. Kerov S., Okounkov A., Olshanski G. The boundary of Young graph with Jack edge multiplicities. Intern. Math. Res. Notices, 4, 173-199 (1998) (preprint version: http: //xxx.lanl.gov/abs/q-alg/9703037).

6. Kerov S., Olshanski G., Vershik A. Harmonic analysis on the infinite symmetric group. A deformation of the regular representation. C. R. Acad. Sci. Paris, Sér. I, 316, 773778 (1993).

7. Macdonald I. G. Symmetric functions and Hall polynomials. 2nd edition, Oxford University Press, 1995.

8. Ольшанский Г. И. Унитарные представления $(G, K)$-пар, связанных с бесконечной симметрической группой $S(\infty)$. Алгебра и анализ, 1, вып. 4, 178-209 (1989).

9. Olshanski $G$. Point processes and the infinite symmetric group. Part I: The general formalism and the density function. Preprint, 1998, http://xxx.lanl.gov/abs/ math/9804086.

10. Borodin A. Point processes and the infinite symmetric group. Part II: Higher correlation functions. Preprint, 1998, http://xxx.lanl.gov/abs/math/9804087.

11. Borodin A., Olshanski G. Point processes and the infinite symmetric group. Part III: Fermion point processes. Preprint, 1998, http://xxx.lanl.gov/abs/math/9804088.

12. Borodin $A$. Point processes and the infinite symmetric group. Part IV: Matrix Whittaker kernel. Preprint, 1998, http://xxx.lanl.gov/abs/math/9810013.

13. Olshanski $G$. Point processes and the infinite symmetric group. Part V: Analysis of the matrix Whittaker kernel. Preprint, 1998, http://xxx.lanl.gov/abs/math/9810014.

14. Thoma E. Die unzerlegbaren, positive-definiten Klassenfunktionen der abzählbar unendlichen, symmetrischen Gruppe. Math. Z., 85, 40-61 (1964). 